\title{
Sequential communication with ex post participation constraints
}

\author{
Stefan Ambec*
}

\author{
INRA-GAEL, University of Grenoble, \\ BP 47, 38040 Grenoble Cedex 9, France, \\ e-mail: ambec@grenoble.inra.fr,
} phone: 33476828336 , fax: 33476825455 .

January 2007

\footnotetext{
${ }^{*}$ I thank Vianney Dequiedt, Patrick González, Ulrich Hege, Rafael Repullo, Masako Ueda, an associate editor and two referees for helpful comments on an early version. This paper was initiated when I was TMR fellow at CSEF, University of Salerno, Italy. I acknowledge financial support from the RTN program "Understanding Financial Architecture: Legal and Political Framework and Economic Efficiency" from the European Commission and the Consiglio Nazionale delle Ricerche (CNR), Italy.
} 


\begin{abstract}
This paper examines an informed principal-agent game with ex post participation constraints for the agent. It shows that the players do not lose by communicating in turn among themselves rather than simultaneously if and only if the principal communicates first. It then considers every Bayesian incentive compatible allocation rules that assign nonnegative payoffs for one player in a bilateral asymmetric information framework. It provides necessary and sufficient conditions for sequential communication to be as efficient as simultaneous communication in implementing these allocation rules when the player with unbounded payoffs moves first.
\end{abstract}

Key words: asymmetric information, principal-agent, implementation, contract theory.

JEL codes: D23, D82. 


\section{Introduction}

Consider two agents. They have to select a decision through a mechanism. Preferences are private information. Side-payments are allowed. One of the two agents has limited liability. Which mechanism should they use? According to the Revelation Principal, no mechanism Pareto dominates (in term of ex ante payoffs) a direct revelation mechanism. It prescribes that the agents simultaneously send a message (about preferences) to an arbitrator who then selects a decision (with a side-payment). This paper investigates whether simultaneous communication can be replaced by sequential communication (in which agents communicate in turn among themselves) in direct revelation mechanisms and what such a sequential mechanism look like.

The agents might be the members of an organization who decide on a project size. Or two firms, a producer and a retailer, that contract on production levels and prices. In those examples, the agents are free to quit the organization or to exit the contract, anytime (e.g. by going bankrupt). They are protected by limited liability. Payoffs must therefore be non-negative to insure participation. ${ }^{1}$ Furthermore, by defining who decides first on what, the hierarchical structure in organizations, or the assignment of decision rights in contracts specifies a particular sequence of moves. This paper rationalizes the use of a sequential decision-making process. It also say something about its design.

With simultaneous communication, the allocations implemented (decisions and sidepayments) must be Bayesian incentive compatible (BIC) for both agents. In contrast, with sequential communication, they must satisfy the stronger dominant strategy incentive compatible (DSIC) condition for the agent who communicates last. The paper provides necessary and sufficient conditions for which the DSIC condition for the agent who has limited liability can be obtained for free (in term of expected payoffs). Since, as argued by Crémer and Riordan [1], the DSIC condition has simpler informational and computational requirements, a sequential mechanism is particularly appropriate for dealing with less informed or rationally bounded agents.

The first part of the paper focuses on the allocations implemented if one player, the

\footnotetext{
${ }^{1}$ See Sappington [8] for a discussion on the limited liability assumption in the principal-agent model.
} 
"principal", makes a take-it-or-leave-it contract offer to the other player, the "agent", whose outside option is nil. It shows that simultaneous communication can be replaced by sequential communication if and only if the principal communicates first. This result also holds if the principal has bounded payoffs.

The second part considers all allocation rules that are BIC for both partners and satisfy the ex post participation constraints for one. ${ }^{2}$ It provides necessary and sufficient conditions for which the stronger DSIC condition for the ex post participation constrained partner can be obtained for free. It therefore yields sufficient conditions for the use of sequential mechanisms in place of simultaneous mechanisms while leaving the two partners' expected utility unchanged.

The first part builds on Maskin and Tirole [5]. They show that the principal does not lose by revealing her type before the agent does, thereby imposing the stronger DSIC condition on the agent. ${ }^{3}$ These authors impose individual rationality at the interim stage but not ex post. Here I take the argument one step further by showing that (i) the result also holds when participation is an issue ex post, and (ii) in this case, the sequence of communication matters: the principal can communicate first but not last. In other words, imposing the DSIC condition in place of the BIC condition on the principal reduces her ex ante payoffs.

The second part is related to literature on the implementation of Bayesian mechanisms with quasi-linear preferences. Mookherjee and Reichelstein [7] show that, under sufficient conditions, the BIC constraints can be replaced by the DSIC constraints for all agents while leaving unchanged every agent's expected payoff. Yet this requires alterations to side-payments which affects payoffs. The present paper shows that, on the one hand, some agent's payoffs might be negative. The DSIC condition might therefore not be compatible with ex post participation constraints. ${ }^{4}$ On the other hand, with only two players, one

\footnotetext{
${ }^{2}$ I.e. not necessarily the ones that emerge from an ex ante negotiation with extreme bargaining powers.

${ }^{3}$ To be precise, Maskin and Tirole show that, in their adverse selection model but with quasi-linear preferences, the informed principal does not loose by revealing her type to the agent at the contracting stage, thereby moving incentive compatibility constraints from the interim to the ex post stage (Proposition 11 in their paper).

${ }^{4}$ Proposition 1 shows that this is indeed the case for the allocation implemented in the informed principal-
} 
being ex post participation constrained, BIC for all can be replaced by BIC for one agent and DSIC for the other. ${ }^{5}$

Crémer and Riordan [1] show that, under sufficient conditions, BIC for all can be replaced by BIC for one agent and DSIC for the others. However, they do not address the participation or individual rationality issue. ${ }^{6}$ Here, I show that (i) this result might also hold with two agents when participation is an issue ex post for one agent; and (ii) it matters who is DSIC constrained and who is BIC constrained.

\section{Model and definitions}

Take two agents, hereafter called "players", a principal $P$ ("she") and an agent $A$ ("he"). Each player $i=P, A$ has preferences $u_{i}\left(y, \theta_{i}\right)$ over a common decision $y \in \mathbb{Y} \subseteq \mathbb{R}$ and private information $\theta_{i} \in\left[\underline{\theta}_{i}, \bar{\theta}_{i}\right] \equiv \Theta_{i}$, with $\underline{\theta}_{i}<\bar{\theta}_{i}$. $\theta_{i}$ is referred as $i$ 's type. It is commonknowledge that $\theta_{P}$ and $\theta_{A}$ are independently distributed according to the density $f_{i}\left(\theta_{i}\right)>0$ for every $\theta_{i} \in \Theta_{i}$ and cumulative $F_{i}\left(\theta_{i}\right)$ for $i=P, A$. The function $u_{i}$ is assumed thrice continuously differentiable, concave in $y$, and increasing in $\theta_{i}$. I make the assumption of increasing marginal utility with $\theta_{i}$ for every $i=P, A$, known as the "single-crossing property". ${ }^{7}$

$$
\text { (A1) } \frac{\partial^{2} u_{i}}{\partial y \partial \theta_{i}}>0
$$

Players perform transfers among themselves. The net transfer (possibly negative) from the principal to the agent is denoted $t$. An allocation is a vector $(y, t)$. The principal $P$ and the agent $A$ 's payoffs with the allocation $(y, t)$ in state $\left(\theta_{P}, \theta_{A}\right) \in \Theta_{P} \times \Theta_{A}$ are, respectively:

$$
U_{P}\left(y, t, \theta_{P}\right) \equiv u_{P}\left(y, \theta_{P}\right)-t
$$

agent game.

${ }^{5}$ Proposition 2 provides the conditions for which BIC for all can be replaced by BIC for one partner and DSIC for the other.

${ }^{6}$ In particular, they focus on the Bayesian implementation of the first-best levels of public good which might not be compatible with the agents' participation condition (see, e.g., Example 23.E.1. in Mas-Colell et al. [4]).

${ }^{7}$ This condition ensures that a solution derived by recognizing only the "local" incentive constraints will also be globally incentive compatible. 
and,

$$
U_{A}\left(y, t, \theta_{A}\right) \equiv u_{A}\left(y, \theta_{A}\right)+t
$$

A state of nature is a vector $\boldsymbol{\theta}=\left(\theta_{P}, \theta_{A}\right) \in \Theta_{P} \times \Theta_{A} \equiv \boldsymbol{\Theta}$. The first component of $\boldsymbol{\theta}$ is the principal's private information while the second is the agent's one. The (total) surplus or profit in state $\boldsymbol{\theta}$ is:

$$
\pi(y, \boldsymbol{\theta})=U_{P}\left(y, t, \theta_{P}\right)+U_{A}\left(y, t, \theta_{A}\right)=u_{P}\left(y, \theta_{P}\right)+u_{A}\left(y, \theta_{A}\right) .
$$

It is assumed to be non-negative, strictly concave and increasing up to its maximum $y^{*}(\boldsymbol{\theta}) \in$ $\mathbb{Y}$ defined by the only value satisfying $\frac{\partial \pi}{\partial y}\left(y^{*}(\boldsymbol{\theta}), \boldsymbol{\theta}\right)=0$. Given the above assumptions, the profit $\pi$ and the marginal profit $\frac{\partial \pi}{\partial y}$ are both increasing with $\theta_{i}$ for $i=P, A$.

Examples of such functions include firm's profits, production costs, revenues from marketing a product, as well as utility functions (e.g. benefits from consuming a level of public good $y)$. For instance, $u_{P}\left(y, \theta_{P}\right)$ might stand for the revenue from marketing $y$ units with a demand level $\theta_{P}$ (e.g. $\left.u_{P}\left(y, \theta_{P}\right)=\left(\theta_{P}-y\right) y\right)$ or a price $\theta_{P}$ (then $\left.u_{P}\left(y, \theta_{P}\right)=\theta_{P} y\right)$ while $u_{A}\left(y, \theta_{A}\right)=-c\left(y, \theta_{A}\right)$ where $c$ is a production cost function with marginal cost decreasing with $\theta_{A}$.

Three stages can be defined to evaluate player's payoffs: ex ante, before players have received any private information; interim, when each player $i$ has received his or her private information $\theta_{i}$ but does not know the other's information; ex post, when the state $\boldsymbol{\theta}$ is public. The corresponding ex ante, interim and ex post payoffs are, respectively, $E_{\boldsymbol{\theta}}\left[U_{i}\left(y\left(\theta_{i}, \theta_{j}\right), t\left(\theta_{i}, \theta_{j}\right), \theta_{i}\right)\right], E_{\theta_{j}}\left[U_{i}\left(y\left(\theta_{i}, \theta_{j}\right), t\left(\theta_{i}, \theta_{j}\right), \theta_{i}\right)\right]$, and $U_{i}\left(y\left(\theta_{i}, \theta_{j}\right), t\left(\theta_{i}, \theta_{j}\right), \theta_{i}\right)$ for $i=P, A, j \neq i .^{8}$

An allocation rule $\{y(\boldsymbol{\theta}), t(\boldsymbol{\theta})\}$ is a menu of allocations $(y(\boldsymbol{\theta}), t(\boldsymbol{\theta}))$ contingent on each state of nature $\boldsymbol{\theta} \in \boldsymbol{\Theta}$.

Definition 1 The allocation rule $\{y(\boldsymbol{\theta}), t(\boldsymbol{\theta})\}$ is Bayesian incentive-compatible (BIC) for $i$ if

$$
\theta_{i} \in \arg \max _{\tilde{\theta}_{i}} E_{\theta_{j}}\left[U_{i}\left(y\left(\tilde{\theta}_{i}, \theta_{j}\right), t\left(\tilde{\theta}_{i}, \theta_{j}\right), \theta_{i}\right)\right]
$$

\footnotetext{
${ }^{8}$ The terminology is similar to Holmström and Maskin [3]. $E_{\theta_{j}}\left(E_{\boldsymbol{\theta}}\right)$ denotes the expectation operator over $\theta_{j}(\boldsymbol{\theta})$.
} 
for every $\theta_{i} \in \Theta_{i}, \theta_{j} \in \Theta_{j}$, and for $j \neq i$.

Definition 2 The allocation rule $\{y(\boldsymbol{\theta}), t(\boldsymbol{\theta})\}$ is dominant strategy incentive-compatible (DSIC) for $i$ if

$$
\theta_{i} \in \arg \max _{\tilde{\theta}_{i}} U_{i}\left(y\left(\tilde{\theta}_{i}, \theta_{j}\right), t\left(\tilde{\theta}_{i}, \theta_{j}\right), \theta_{i}\right)
$$

for every $\theta_{i} \in \Theta_{i}, \theta_{j} \in \Theta_{j}$, and for $j \neq i$.

The conditions in Definition 1 (Definition 2) are the standard incentive-compatible constraints that allocation rules must satisfy in any direct revelation mechanism in a Bayesian (dominant) strategy equilibrium. Bayesian incentive-compatibility (dominant strategy incentive-compatibility) requires that truthful reporting of private information maximizes the player's interim (ex post) payoff. Of course, dominant strategy incentive-compatibility is a stronger requirement in the sense that any DSIC allocation rule is BIC for $i$ but the reverse is not necessarily true.

In the rest of the paper I will often use the following equivalent formulations of Bayesian incentive-compatibility and dominant strategy incentive compatibility.

Lemma 1 The allocation rule $\{y(\boldsymbol{\theta}), t(\boldsymbol{\theta})\}$ is BIC for $i$ if and only if:

(i) $E_{\theta_{j}}\left[y\left(., \theta_{j}\right)\right]$ is non-decreasing,

(ii) $E_{\theta_{j}}\left[U_{i}\left(y\left(\theta_{i}, \theta_{j}\right), t\left(\theta_{i}, \theta_{j}\right), \theta_{i}\right)\right]=$

$$
\underbrace{\int_{\underline{\theta}_{i}}^{\theta_{i}} E_{\theta_{j}}\left[\frac{\partial u_{i}}{\partial \theta_{i}}\left(y\left(x, \theta_{j}\right), x\right) d x\right]}_{(a)}+\underbrace{E_{\theta_{j}}\left[U_{i}\left(y\left(\underline{\theta}_{i}, \theta_{j}\right), t\left(\underline{\theta}_{i}, \theta_{j}\right), \underline{\theta}_{i}\right)\right]}_{(b)}
$$

for every $\theta_{i} \in \Theta_{i}, \theta_{j} \in \Theta_{j}$, and for $j \neq i$.

Lemma 2 The allocation rule $\{y(\boldsymbol{\theta}), t(\boldsymbol{\theta})\}$ is DSIC for $i$ if and only if:

(i) $y\left(., \theta_{j}\right)$ is non-decreasing,

(ii) $U_{i}\left(y\left(\theta_{i}, \theta_{j}\right), t\left(\theta_{i}, \theta_{j}\right), \theta_{i}\right)=\underbrace{\int_{\theta_{i}}^{\theta_{i}} \frac{\partial u_{i}}{\partial \theta_{i}}\left(y\left(x, \theta_{j}\right), x\right) d x}_{(a)}+\underbrace{U_{i}\left(y\left(\underline{\theta}_{i}, \theta_{j}\right), t\left(\underline{\theta}_{i}, \theta_{j}\right), \underline{\theta}_{i}\right)}_{(b)}$, 
for every $\theta_{i} \in \Theta_{i}, \theta_{j} \in \Theta_{j}$, and for $j \neq i$.

Lemmata 1 and 2 set out well-known results in mechanism design, mostly due to Mirrless [6], in the context of the present model. Formal proofs can be found in Fudenberg and Tirole [2] and Mas-Colell et al. [4]. Although the second conditions in the above lemma appear somewhat complex, they have a straightforward interpretation. Condition (ii) in Lemma 1 (Lemma 2) breaks down $i$ 's interim (ex post) payoff into two terms. The second term (b) is $i$ 's interim (ex post) payoff when of type $\underline{\theta}_{i}$. The first term (a) is the incremental interim (ex post) benefit of a higher type $\theta_{i}>\underline{\theta}_{i}$. Condition (ii) stipulates that $i$ must receive exactly the incremental benefit of reporting truthfully $\theta_{i}$.

Definition 3 The allocation rule $\{y(\boldsymbol{\theta}), t(\boldsymbol{\theta})\}$ satisfies ex post participation (or limited liability) for $i$ if and only if $i$ 's ex post payoffs are non-negative, i.e. $U_{i}\left(y(\boldsymbol{\theta}), t(\boldsymbol{\theta}), \theta_{i}\right) \geq$ 0 for every $\boldsymbol{\theta} \in \mathbf{\Theta}$.

A limited-liability incentive rule is an allocation rule that is BIC for both players and satisfies ex post participation for the agent. This paper examines the equivalent implementation of incentive allocation rules with sequential communication in the sense defined below.

Definition 4 A limited-liability incentive rule can equivalently be implemented in a sequential mechanism (or with sequential communication) in which $i$ communicates first if there exists an allocation rule which is:

(i) BIC for $i$,

(ii) DSIC for $j \neq i$,

(iii) It satisfies the agent's ex-post participation constraints,

(iv) It yields the same ex ante payoff to both players as does the limited-liability incentive rule.

To be equivalently implemented in a sequential mechanism, a limited-liability incentive rule must satisfy the stronger requirement of dominant strategy incentive-compatibility for one 
player. The sequence of communication determines the identity of this player. Once $i$ has communicated her or his type honestly, $j \neq i$ selects her or his reporting strategy under perfect information. Therefore, $j$ 's incentive-compatible constraints must be satisfied ex post in every state of nature, i.e., in dominant strategy.

I first focus on specific limited-liability incentive rules: those that are implemented in the informed principal-agent game defined in the next section. I consider all limited-liability incentive rules in Section 4.

\section{Sequential communication in the informed principal-agent game}

Consider the following benchmark game. Suppose that the principal makes an ex ante take-it-or-leave-it limited-liability incentive rule offer $\{y(\boldsymbol{\theta}), t(\boldsymbol{\theta})\}$ to the agent. If it is refused, each player gets zero. If it is accepted, the game proceeds. Each player $i$ privately observes $\theta_{i}$. Players simultaneously send direct messages $\hat{\theta}_{i}$ which select a single allocation $\left(y\left(\hat{\theta}_{P}, \hat{\theta}_{A}\right), t\left(\hat{\theta}_{P}, \hat{\theta}_{A}\right)\right)$ in the limited-liability incentive rule.

The limited-liability incentive rules implemented in a Perfect Bayesian Nash Equilibrium of the benchmark game, denoted $\left\{y^{P}(\boldsymbol{\theta}), t^{P}(\boldsymbol{\theta})\right\}$, are those that maximize the principal's ex ante payoff. Although several transfer rules $\left\{t^{P}(\boldsymbol{\theta})\right\}$ can be a solution, the decision rule $\left\{y^{P}(\boldsymbol{\theta})\right\}$ is unique and easy to characterize under some assumptions. To do that, we need first to find out the agent's ex ante payoff. From (ii) in Lemma 1, taking expectation with respect to $\theta_{A}$ yields $E_{\boldsymbol{\theta}}\left[U_{A}\left(y\left(\theta_{P}, \theta_{A}\right), t\left(\theta_{P}, \theta_{A}\right), \theta_{A}\right)\right]=$

$$
E_{\boldsymbol{\theta}}\left[\int_{\underline{\theta}_{A}}^{\theta_{A}} \frac{\partial u_{A}}{\partial \theta_{A}}\left(y\left(\theta_{P}, x\right), x\right) d x\right]+E_{\boldsymbol{\theta}}\left[U_{A}\left(y\left(\theta_{P}, \underline{\theta}_{A}\right), t\left(\theta_{P}, \underline{\theta}_{A}\right), \underline{\theta}_{A}\right)\right] .
$$

Integrating by parts the expectation with respect to $\theta_{A}$ in the first term yields

$$
\begin{aligned}
E_{\boldsymbol{\theta}}\left[U_{A}\left(y\left(\theta_{P}, \theta_{A}\right), t\left(\theta_{P}, \theta_{A}\right), \theta_{A}\right)\right]= \\
\underbrace{E_{\boldsymbol{\theta}}\left[\frac{1-F_{A}\left(\theta_{A}\right)}{f_{A}\left(\theta_{A}\right)} \frac{\partial u_{A}}{\partial \theta_{A}}\left(y\left(\theta_{P}, \theta_{A}\right), \theta_{A}\right)\right]}_{(a)}+\underbrace{E_{\boldsymbol{\theta}}\left[U_{A}\left(y\left(\theta_{P}, \underline{\theta}_{A}\right), t\left(\theta_{P}, \underline{\theta}_{A}\right), \underline{\theta}_{A}\right)\right]}_{(b)} .
\end{aligned}
$$


The agent's ex ante payoff is broken down into two terms. The first term (a) is his expected informational rent. It is the minimal amount that provides him with incentives to communicate truthfully. The second term (b) is the agent of type $\underline{\theta}_{A}$ 's interim payoff. The principal sets this term equal to zero to maximize her ex ante payoff while satisfying the BIC and ex post participation constraints. Therefore, the agent's ex ante payoff is just (a). To simplify the notation, denote $r_{A}\left(y, \theta_{A}\right) \equiv \frac{1-F_{A}\left(\theta_{A}\right)}{f_{A}\left(\theta_{A}\right)} \frac{\partial u_{A}}{\partial \theta_{A}}\left(y, \theta_{A}\right)$ so that the agent's ex ante payoff becomes $E_{\boldsymbol{\theta}}\left[r_{A}\left(y\left(\theta_{P}, \theta_{A}\right), \theta_{A}\right)\right]$. Due to (1), the principal's ex ante payoff is

$$
E_{\boldsymbol{\theta}}\left[\pi\left(y\left(\theta_{P}, \theta_{A}\right),\left(\theta_{P}, \theta_{A}\right)\right)-r_{A}\left(y\left(\theta_{P}, \theta_{A}\right), \theta_{A}\right)\right] .
$$

The decision rule implemented $\left\{y^{P}(\boldsymbol{\theta})\right\}$ maximizes (2). Under some assumptions (specified later), it is uniquely defined by the following first order conditions:

$$
\frac{\partial \pi}{\partial y}\left(y^{P}\left(\theta_{P}, \theta_{A}\right),\left(\theta_{P}, \theta_{A}\right)\right)-\frac{\partial r_{A}}{\partial y}\left(y^{P}\left(\theta_{P}, \theta_{A}\right), \theta_{A}\right)=0,
$$

for all $\left(\theta_{P}, \theta_{A}\right) \in \boldsymbol{\Theta}$. The decision scheme $\left\{y^{P}(\boldsymbol{\theta})\right\}$ solves a trade-off between maximizing the total surplus and minimizing the agent's informational rent. As a consequence, except "at the top" (for the agent), decisions are distorted downward: $y^{P}\left(\theta_{P}, \theta_{A}\right)<y^{*}\left(\theta_{P}, \theta_{A}\right)$ for all $\left(\theta_{P}, \theta_{A}\right)$ such that $\theta_{A} \neq \bar{\theta}_{A}$. The second-order conditions are satisfied under the following assumption.

$$
\text { (A2) } \frac{\partial^{3} u_{A}}{\partial y^{2} \partial \theta_{A}} \geq 0
$$

Totally differentiating (3) with respect to $\theta_{A}$ shows that the requirement of $y^{P}\left(\theta_{P},.\right)$ nondecreasing holds under the following supplementary assumptions. ${ }^{9}$

$$
\begin{aligned}
& \text { (A3) } \frac{\partial^{3} u_{A}}{\partial y \partial^{2} \theta_{A}} \leq 0 . \\
& \text { (A4) } \frac{d}{d \theta_{A}}\left[\frac{1-F_{A}\left(\theta_{A}\right)}{f_{A}\left(\theta_{A}\right)}\right] \leq 0 .
\end{aligned}
$$

\footnotetext{
${ }^{9}$ Both assumptions imply that $\frac{\partial^{2} r_{A}}{\partial y \partial \theta_{A}}\left(y^{P}\left(\theta_{P}, \theta_{A}\right), \theta_{A}\right) \leq 0$ for every $\left(\theta_{P}, \theta_{A}\right) \in \boldsymbol{\Theta}$ which ensures that $y^{P}\left(\theta_{P},.\right)$ is increasing for all $\theta_{P}$. (A1) and (A2) and the concavity of $\pi(., \boldsymbol{\theta})$ imply that $y^{P}\left(., \theta_{A}\right)$ is increasing.
} 
Assumption (A4) states that the "hazard rate" $\frac{f_{A}\left(\theta_{A}\right)}{1-F_{A}\left(\theta_{A}\right)}$ is non-decreasing. It is satisfied for instance if the density $f_{A}$ is non-decreasing. Assumptions (A1) to (A4) are standard in mechanism design (e.g. Fudenberg and Tirole [2], Chapter 7). Armed with those assumptions, we set out a first result.

Proposition 1 Under assumptions (A1) to (A4), the limited-liability incentive rule implemented in the informed principal-agent game is equivalently implementable in a sequential mechanism if and only if the principal communicates first.

Proof (If part) Consider the following transfer scheme:

$$
\tilde{t}\left(\theta_{P}, \theta_{A}\right)=-u_{A}\left(y^{P}\left(\theta_{P}, \theta_{A}\right), \theta_{A}\right)+\int_{\underline{\theta}_{A}}^{\theta_{A}} \frac{\partial u_{A}}{\partial \theta_{A}}\left(y^{P}\left(\theta_{P}, x\right), x\right) d x .
$$

It yields $U_{A}\left(y^{P}\left(\theta_{P}, \theta_{A}\right), \tilde{t}\left(\theta_{P}, \theta_{A}\right), \theta_{A}\right)=\int_{\underline{\theta}_{A}}^{\theta_{A}} \frac{\partial u_{A}}{\partial \theta_{A}}\left(y^{P}\left(\theta_{P}, x\right), x\right) d x$. First, since it is nonnegative for every $\theta \in \Theta$, then the ex post participation constraints hold. Second, by construction it satisfies condition (ii) in Lemma 2. Moreover, since $y^{P}\left(\theta_{P},.\right)$ is nondecreasing for every $\theta_{P} \in \Theta_{P}$, then condition (i) in Lemma 2 is also satisfied and, therefore, the agent's DSIC constraints hold. The above transfer scheme yields to the principal $E_{\theta_{A}}\left[U_{P}\left(y^{P}\left(\theta_{P}, \theta_{A}\right), \tilde{t}\left(\theta_{P}, \theta_{A}\right), \theta_{P}\right)\right]=E_{\theta_{A}}\left[\pi\left(y^{P}\left(\theta_{P}, \theta_{A}\right),\left(\theta_{P}, \theta_{A}\right)\right)-r_{A}\left(y^{P}\left(\theta_{P}, \theta_{A}\right), \theta_{A}\right)\right]$ at the interim stage. Since $y^{P}\left(\theta_{P}, \theta_{A}\right)$ maximizes $\pi\left(y\left(\theta_{P}, \theta_{A}\right),\left(\theta_{P}, \theta_{A}\right)\right)-r_{A}\left(y\left(\theta_{P}, \theta_{A}\right), \theta_{A}\right)$ for every $\left(\theta_{P}, \theta_{A}\right) \in \boldsymbol{\Theta}$, then $\theta_{P}$ maximizes $E_{\theta_{A}}\left[\pi\left(y^{P}\left(\hat{\theta}_{P}, \theta_{A}\right),\left(\theta_{P}, \theta_{A}\right)\right)-r_{A}\left(y^{P}\left(\hat{\theta}_{P}, \theta_{A}\right), \theta_{A}\right)\right]$ with respect to $\hat{\theta}_{P}$ and, therefore, the allocation rule $\left\{y^{P}(\boldsymbol{\theta}), \tilde{t}(\boldsymbol{\theta})\right\}$ is BIC for the principal.

(Only if part) First, I show that any allocation rule that equivalently implements $\left\{y^{P}(\boldsymbol{\theta}), t^{P}(\boldsymbol{\theta})\right\}$ must assign zero ex post payoffs to the agent of type $\underline{\theta}_{A}$. Suppose it is not the case. Suppose that an allocation rule that equivalently implements $\left\{y^{P}(\boldsymbol{\theta}), t^{P}(\boldsymbol{\theta})\right\}$ assigns a strictly positive ex post payoff to the agent in say state $\left(\theta_{P}^{\prime}, \underline{\theta}_{A}\right)$. Then, due to the ex post participation constraints, the agent of type $\underline{\theta}_{A}$ 's interim payoff is also strictly positive. Using the definition of the BIC conditions for the agent in Lemma 1, applying the expectation operator on $\theta_{A}$ and integrating by parts as before, shows that the agent's ex ante payoff is then strictly higher than $E_{\boldsymbol{\theta}}\left[r_{A}\left(y(\boldsymbol{\theta}), \theta_{A}\right)\right]$. Therefore, ex ante, the principal obtains strictly less than the maximal value of $E_{\boldsymbol{\theta}}\left[\pi(y(\boldsymbol{\theta}), \boldsymbol{\theta})-r_{A}\left(y(\boldsymbol{\theta}), \theta_{A}\right)\right]$ obtained 
with simultaneous communication which contradicts that the allocation rule equivalently implements $\left\{y^{P}(\boldsymbol{\theta}), t^{P}(\boldsymbol{\theta})\right\}$.

Second, I show that any limited-liability incentive rule that yields zero ex post payoffs to the agent of type $\underline{\theta}_{A}$ violates the principal's DSIC constraints. Since the agent gets nothing in states $\left(\theta_{P}, \underline{\theta}_{A}\right)$ for every $\theta_{P} \in \Theta_{P}$, the principal obtains the surplus, namely $\pi\left(y^{P}\left(\theta_{P}, \underline{\theta}_{A}\right),\left(\theta_{P}, \underline{\theta}_{A}\right)\right)$, in those states. Since $y^{P}\left(\theta_{P}, \underline{\theta}_{A}\right)<y^{*}\left(\theta_{P}, \underline{\theta}_{A}\right)$ for every $\theta_{P} \in \Theta_{P}$, then there exists a type $\hat{\theta}_{P}>\underline{\theta}_{P}$ such that $\pi\left(y^{P}\left(\hat{\theta}_{P}, \underline{\theta}_{A}\right),\left(\theta_{P}, \underline{\theta}_{A}\right)\right)>\pi\left(y^{P}\left(\theta_{P}, \underline{\theta}_{A}\right),\left(\theta_{P}, \underline{\theta}_{A}\right)\right)$ for any $\theta_{P} \in \Theta_{P}$, which implies that the principal prefers to send the message $\hat{\theta}_{P}$ rather than revealing truthfully $\theta_{P} \cdot{ }^{10}$

Proposition 1 posits that, in the context of an informed principal-agent game with an agent ex post participation constrained, there is no loss of efficiency if communication occurs sequentially if and only if the principal communicates first.

In the principal-agent model, the principal gets the ex ante surplus net of the agent's expected informational rent. One way to achieve this ex ante payoff while satisfying the agent's DSIC and ex post participation constraints is to assign to the agent his ex post incremental gain of reporting truthfully his type (the term (a) of part (ii) in Lemma 2). With such an allocation rule, the principal obtains the total surplus net of the agent's informational rent not only at the ex ante but also at the interim stage. When sending the message $\hat{\theta}_{P}$, the principal selects a subset of decisions $\left\{y^{P}\left(\hat{\theta}_{P}, \theta_{A}\right)\right\}_{\theta_{A} \in \Theta_{A}}$ in the set $\left\{y^{P}(\boldsymbol{\theta})\right\}$. She would obviously select the one that maximizes her interim payoff when of type $\theta_{P}$ which is $\left\{y^{P}\left(\theta_{P}, \theta_{A}\right)\right\}_{\theta_{A} \in \Theta_{A}}$. She therefore reports truthfully her type $\theta_{P}$. In other words, the allocation rule is BIC for the principal.

On the other hand, to satisfy the agent's BIC and ex post participation constraints, the limited-liability incentive rule must assign to the agent the incremental gain of reporting truthfully his type at the interim stage, i.e., the term (a) of part (ii) in Lemma 1. The principal gets the surplus net of this rent. She therefore obtains all the surplus when the agent's type is $\underline{\theta}_{A}$. Since decisions are distorted downward (and assuming that the agent

\footnotetext{
${ }^{10}$ Notice that the argument holds for a measurable set around $\underline{\theta}_{A}$.
} 
reports truthfully $\underline{\theta}_{A}$ ), she prefers to report a higher type ex post. ${ }^{11}$ Hence, the incentive allocation rule cannot be BIC for the agent, DSIC for the principal while assigning nonnegative payoffs to the agent.

For example, suppose that $u_{P}\left(y, \theta_{P}\right)=\left(\theta_{P}-y\right) y$ and $u_{A}\left(y, \theta_{A}\right)=-\left(\alpha-\theta_{A}\right) y$ with $\underline{\theta}_{P}+\underline{\theta}_{A}>\alpha>\bar{\theta}_{A}$. Suppose further that $\theta_{A}$ is uniformly distributed in $\Theta_{A}$. Then $\pi(y, \theta)=\left(\theta_{P}+\theta_{A}-\alpha-y\right) y$ is maximized at $y^{*}\left(\theta_{P}, \theta_{A}\right)=\frac{1}{2}\left(\theta_{P}+\theta_{A}-\alpha\right)$. The principal's maximization objective in $(2)$ is $\left(\theta_{P}+2 \theta_{A}-\alpha-\bar{\theta}_{A}-y\right) y$. It is maximized at $y^{P}\left(\theta_{P}, \theta_{A}\right)=\frac{1}{2}\left(\theta_{P}+2 \theta_{A}-\alpha-\bar{\theta}_{A}\right)$, which is strictly lower than $y^{*}\left(\theta_{P}, \theta_{A}\right)$, except "at the top" $\bar{\theta}_{A}$. To achieve the principal's ex ante payoff in the benchmark game while satisfying the agent's BIC and ex post participation constraints, the agent of type $\underline{\theta}_{A}$ 's ex post payoffs must be nil. It implies that the principal must obtain all surplus when the agent is of type $\underline{\theta}_{A}$ whatever her type is. If she communicates last, the principal chooses $\hat{\theta}_{P}$ that maximizes is ex post payoff $\pi\left(y^{P}\left(\hat{\theta}_{P}, \underline{\theta}_{A}\right),\left(\theta_{P}, \underline{\theta}_{A}\right)\right)=\frac{1}{4}\left(2 \theta_{P}-\hat{\theta}_{P}-\alpha+\bar{\theta}_{A}\right)\left(\hat{\theta}_{P}+2 \underline{\theta}_{A}-\alpha-\bar{\theta}_{A}\right)$. Therefore, she reports $\hat{\theta}_{P}=\theta_{P}+\bar{\theta}_{A}-\underline{\theta}_{A}>\theta_{P}$ for every $\theta_{P} \in \Theta_{P}$.

As a next step, I further investigate the informed principal-agent game, imposing nonnegative payoffs for the principal as well. It is easy to show that, if $u_{A}$ is concave in $\theta_{A}$, then the allocation rule $\left\{y^{P}(\boldsymbol{\theta}), \tilde{t}(\boldsymbol{\theta})\right\}$ (which is defined in (3) and (4)) assigns non-negative ex post payoffs to the principal. Indeed, the principal obtains in state $\boldsymbol{\theta}$ :

$$
U_{P}\left(y^{P}(\boldsymbol{\theta}), \tilde{t}(\boldsymbol{\theta}), \theta_{P}\right)=\pi\left(y^{P}(\boldsymbol{\theta}), \boldsymbol{\theta}\right)-\int_{\underline{\theta}_{A}}^{\theta_{A}} \frac{\partial u_{A}}{\partial \theta_{A}}\left(y^{P}\left(\theta_{P}, x\right), x\right) d x .
$$

Since $\pi\left(y^{P}\left(\theta_{P}, \underline{\theta}_{A}\right),\left(\theta_{P}, \underline{\theta}_{A}\right)\right) \geq 0$ for every $\theta_{P} \in \Theta$, all we have to show is that the righthand term in (5) is non-decreasing in $\theta_{A}$. Differentiate (5) with respect to $\theta_{A}$ and use the envelope theorem to compute:

$$
\frac{\partial \pi}{\partial \theta_{A}}\left(y^{P}(\boldsymbol{\theta}), \boldsymbol{\theta}\right)-\frac{\partial u_{A}}{\partial \theta_{A}}\left(y^{P}(\boldsymbol{\theta}), \theta_{A}\right)-\int_{\underline{\theta}_{A}}^{\theta_{A}} \frac{\partial^{2} u_{A}}{\partial \theta_{A}^{2}}\left(y^{P}\left(\theta_{P}, x\right), x\right) d x .
$$

Since $\frac{\partial \pi}{\partial \theta_{A}}\left(y^{P}(\boldsymbol{\theta}), \boldsymbol{\theta}\right)=\frac{\partial u_{A}}{\partial \theta_{A}}\left(y^{P}(\boldsymbol{\theta}), \theta_{A}\right)$, then the above term is non-negative under the assumption $\frac{\partial^{2} u_{A}}{\partial \theta_{A}^{2}}\left(y^{P}(\boldsymbol{\theta}), \theta_{A}\right) \leq 0$ for all $\boldsymbol{\theta} \in \boldsymbol{\Theta}$.

\footnotetext{
${ }^{11}$ If her type is say $\theta_{P}^{\prime}<\bar{\theta}_{P}$, she prefers to report $\theta_{P}^{\prime \prime}>\theta_{P}^{\prime}$ (ideally $\theta_{P}^{\prime \prime}$ such that $y^{P}\left(\theta_{P}^{\prime \prime}, \underline{\theta}_{A}\right)=y^{*}\left(\theta_{P}^{\prime}, \underline{\theta}_{A}\right)$ if it exists) in order to maximize the surplus which is also her payoff.
} 
Corollary 1 Suppose $u_{A}(y,$.$) is concave for any y \in \mathbb{Y}$, then Proposition 1 also holds when the principal's ex post payoffs are constrained to be non-negative.

Corollary 1 provides a clear link between communication order and bargaining power when both players are treated similarly regarding ex post participation. It states that sequential communication performs as well as simultaneous communication if and only if the player who has bargaining power (the principal) moves first.

\section{Equivalent implementation of limited-liability incentive rules with sequential communication}

In this section I examine the equivalent implementation of any limited-liability incentive rule $^{12}$, i.e. not only the one implemented in the principal-agent game. Indeed other limitedliability incentive rules might be considered. For instance, a regulator might want to implement the ex post efficient decisions $\left\{y^{*}(\boldsymbol{\theta})\right\}$ with a specific divide of the (maximal) ex ante surplus $E_{\boldsymbol{\theta}}\left[\pi\left(y^{*}(\boldsymbol{\theta}), \boldsymbol{\theta}\right)\right]$. Suppose that this can be done with simultaneous communication. Is it possible to rely on a (more decentralized) sequential mechanism?

Notice that, in this section, the "principal" refers to the player with unbounded ex post payoffs. She is privately informed and does necessarily have all the bargaining power.

It is useful to show that the DSIC condition combined with the ex post participation constraints imposes specific lower bounds on the agent's payoff. From (ii) in Lemma 2, taking expectation with respect to $\theta_{A}$ yields, for all $\theta_{P} \in \Theta_{P}, E_{\theta_{A}}\left[U_{A}\left(y\left(\theta_{P}, \theta_{A}\right), t\left(\theta_{P}, \theta_{A}\right), \theta_{A}\right)\right]=$

$$
E_{\theta_{A}}\left[\int_{\underline{\theta}_{A}}^{\theta_{A}} \frac{\partial u_{A}}{\partial \theta_{A}}\left(y\left(\theta_{P}, x\right), x\right) d x\right]+U_{A}\left(y\left(\theta_{P}, \underline{\theta}_{A}\right), t\left(\theta_{P}, \underline{\theta}_{A}\right), \underline{\theta}_{A}\right) .
$$

Integrating by parts the expectation with respect to $\theta_{A}$ in the first term yields, for all $\theta_{P} \in \Theta_{P}$

$$
E_{\theta_{A}}\left[U_{A}\left(y\left(\theta_{P}, \theta_{A}\right), t\left(\theta_{P}, \theta_{A}\right), \theta_{A}\right)\right]=E_{\theta_{A}}\left[r_{A}\left(y\left(\theta_{P}, \theta_{A}\right), \theta_{A}\right)\right]+U_{A}\left(y\left(\theta_{P}, \underline{\theta}_{A}\right), t\left(\theta_{P}, \underline{\theta}_{A}\right), \underline{\theta}_{A}\right) .
$$

\footnotetext{
${ }^{12}$ Recall that limited-liability incentive are allocation rules that are BIC for both players and satisfy ex post participation for the agent.
} 
The ex post participation constraints require the second right-hand term to be non-negative, therefore, for all $\theta_{P} \in \Theta_{P}$,

$$
E_{\theta_{A}}\left[U_{A}\left(y\left(\theta_{P}, \theta_{A}\right), t\left(\theta_{P}, \theta_{A}\right), \theta_{A}\right)\right] \geq E_{\theta_{A}}\left[r_{A}\left(y\left(\theta_{P}, \theta_{A}\right), \theta_{A}\right)\right]
$$

The conditions (6) set lower bounds on the agent's shares of the surplus once $\theta_{P}$ is public information (e.g. after that the principal has truthfully revealed her type $\theta_{P}$ when communicating first). Since the total surplus is divided among the two players (see (1)), these conditions set upper bounds on the principal's interim payoffs, i.e., for all $\theta_{P} \in \Theta_{P}$,

$$
E_{\theta_{A}}\left[U_{P}\left(y\left(\theta_{P}, \theta_{A}\right), t\left(\theta_{P}, \theta_{A}\right), \theta_{P}\right)\right] \leq E_{\theta_{A}}\left[\pi\left(y\left(\theta_{P}, \theta_{A}\right),\left(\theta_{P}, \theta_{A}\right)\right)-r_{A}\left(y\left(\theta_{P}, \theta_{A}\right), \theta_{A}\right)\right]
$$

Notice that, in the principal-agent game, (7) is binding for each $\theta_{P}$. That is precisely why the principal has an incentive to select the subset of decisions $\left\{y^{P}\left(\theta_{P}, \theta_{A}\right)\right\}_{\theta_{A} \in \Theta_{A}}$ that maximizes her interim payoffs, i.e. the right-hand side of (7), by communicating truthfully her type $\theta_{P}$. Yet it might not bind for other divides of the surplus.

It turns out that the above conditions (7) combined with condition (i) of Lemma 2 is not only necessary but also sufficient for the equivalent implementation of an limited-liability incentive rule when the principal moves first. ${ }^{13}$

Proposition 2 Under assumption (A1), a limited-liability incentive rule $\{y(\boldsymbol{\theta}), t(\boldsymbol{\theta})\}$ can be equivalently implemented in a sequential mechanism in which the player with unbounded ex post payoffs communicates first if and only if her interim payoff when of type $\theta_{P}$ does not exceed $E_{\theta_{A}}\left[\pi\left(y\left(\theta_{P}, \theta_{A}\right),\left(\theta_{P}, \theta_{A}\right)\right)-r_{A}\left(y\left(\theta_{P}, \theta_{A}\right), \theta_{A}\right)\right]$ and $y\left(\theta_{P},.\right)$ is non-decreasing for all $\theta_{P} \in \Theta_{P}$.

Proof (If part) Consider a limited-liability incentive rule $\{y(\boldsymbol{\theta}), t(\boldsymbol{\theta})\}$ that satisfies Proposition 2's conditions. Consider further the following transfer scheme for every $\theta \in \Theta$ :

$$
t^{\prime}\left(\theta_{P}, \theta_{A}\right)=-u_{A}\left(y\left(\theta_{P}, \theta_{A}\right), \theta_{A}\right)+\int_{\underline{\theta}_{A}}^{\theta_{A}} \frac{\partial u_{A}}{\partial \theta_{A}}\left(y\left(\theta_{P}, x\right), x\right) d x+\gamma\left(\theta_{P}\right),
$$

with

$$
\gamma\left(\theta_{P}\right) \equiv E_{\theta_{A}}\left[\pi\left(y\left(\theta_{P}, \theta_{A}\right),\left(\theta_{P}, \theta_{A}\right)\right)-r_{A}\left(y\left(\theta_{P}, \theta_{A}\right), \theta_{A}\right)-U_{P}\left(y\left(\theta_{P}, \theta_{A}\right), t\left(\theta_{P}, \theta_{A}\right), \theta_{P}\right)\right] .
$$

\footnotetext{
${ }^{13}$ I thank an anonymous referee for pointing out an error in a previous version of the paper.
} 
First, I show that $\left\{y(\boldsymbol{\theta}), t^{\prime}(\boldsymbol{\theta})\right\}$ is BIC for the principal. Apply the expectation operator over $\theta_{A}$ and use (1) to compute $E_{\theta_{A}}\left[U_{P}\left(y\left(\theta_{P}, \theta_{A}\right), t^{\prime}\left(\theta_{P}, \theta_{A}\right), \theta_{P}\right)\right]=$

$$
E_{\theta_{A}}\left[\pi\left(y\left(\theta_{P}, \theta_{A}\right),\left(\theta_{P}, \theta_{A}\right)\right)-\int_{\underline{\theta}_{A}}^{\theta_{A}} \frac{\partial u_{A}}{\partial \theta_{A}}\left(y\left(\theta_{P}, x\right), x\right) d x\right]-\gamma\left(\theta_{P}\right) .
$$

Integrating by parts yields

$E_{\theta_{A}}\left[U_{P}\left(y\left(\theta_{P}, \theta_{A}\right), t^{\prime}\left(\theta_{P}, \theta_{A}\right), \theta_{P}\right)\right]=E_{\theta_{A}}\left[\pi\left(y\left(\theta_{P}, \theta_{A}\right),\left(\theta_{P}, \theta_{A}\right)\right)-r_{A}\left(y\left(\theta_{P}, \theta_{A}\right), \theta_{A}\right)\right]-\gamma\left(\theta_{P}\right)$.

Substitute for $\gamma\left(\theta_{P}\right)$ and the last relationship simplifies to:

$$
E_{\theta_{A}}\left[U_{P}\left(y\left(\theta_{P}, \theta_{A}\right), t^{\prime}\left(\theta_{P}, \theta_{A}\right), \theta_{P}\right)\right]=E_{\theta_{A}}\left[U_{P}\left(y\left(\theta_{P}, \theta_{A}\right), t\left(\theta_{P}, \theta_{A}\right), \theta_{P}\right)\right]
$$

Therefore, since $\{y(\boldsymbol{\theta}), t(\boldsymbol{\theta})\}$ is BIC for the principal, so is $\left\{y(\boldsymbol{\theta}), t^{\prime}(\boldsymbol{\theta})\right\}$.

Second, I show that $\left\{y(\boldsymbol{\theta}), t^{\prime}(\boldsymbol{\theta})\right\}$ is DSIC for the agent. The agent obtains ex post in each state $\boldsymbol{\theta}$ a payoff

$$
\int_{\underline{\theta}_{A}}^{\theta_{A}} \frac{\partial u_{A}}{\partial \theta_{A}}\left(y\left(\theta_{P}, x\right), x\right) d x+\gamma\left(\theta_{P}\right)
$$

which satisfies condition (ii) in Lemma 2 with $\gamma\left(\theta_{P}\right)=U_{A}\left(y\left(\theta_{P}, \underline{\theta}_{A}\right), t\left(\theta_{P}, \underline{\theta}_{A}\right), \underline{\theta}_{A}\right)$ for all $\theta_{P} \in \Theta_{P}$. Moreover, since $y\left(\theta_{P},.\right)$ is non-decreasing by assumption, it also satisfies condition (i) in Lemma 2.

Third, the agent's ex post payoff in (9) is non-negative for all $\boldsymbol{\theta} \in \boldsymbol{\Theta}$ because $\gamma\left(\theta_{P}\right)$ is non-negative for all $\theta_{P} \in \Theta_{P}$ by assumption (see (7)).

Lastly, (8) shows that $\left\{y(\boldsymbol{\theta}), t^{\prime}(\boldsymbol{\theta})\right\}$ yields the same interim payoff and, therefore, same the ex ante payoff as the limited-liability incentive rule $\{y(\boldsymbol{\theta}), t(\boldsymbol{\theta})\}$ to the principal. Furthermore, (8) combined with (1) yields for every $\theta_{P} \in \Theta_{P}$ :

$$
E_{\theta_{A}}\left[U_{A}\left(y\left(\theta_{P}, \theta_{A}\right), t^{\prime}\left(\theta_{P}, \theta_{A}\right), \theta_{A}\right)\right]=E_{\theta_{A}}\left[U_{A}\left(y\left(\theta_{P}, \theta_{A}\right), t\left(\theta_{P}, \theta_{A}\right), \theta_{A}\right)\right] .
$$

Applying the expectation operator over $\theta_{P}$ shows that $\left\{y(\boldsymbol{\theta}), t^{\prime}(\boldsymbol{\theta})\right\}$ yields the same ex ante payoff to the agent than $\{y(\boldsymbol{\theta}), t(\boldsymbol{\theta})\}$.

(Only if part) The necessary conditions follow from condition (i) in Lemma 2 and the implication of condition (ii) in Lemma 2 established in (6) and then (7). 
The upper bounds (7) on the principal's interim payoffs might be an issue if the principal has outside options at the interim stage. All sequential mechanisms (that equivalently implement a limited-liability incentive rule) might fail to assign to the principal an interim payoff higher than the benefits from the outside options for some types $\theta_{P}$. In contrast, with simultaneous communication, the agent's BIC constraints are in expectation over $\theta_{P}$ and, therefore, do not bound the agent's share of the surplus for any type $\theta_{P}$.

I now close the paper with an example in which the conditions (7) might be a problem. Assume that $y$ is a level of pollution which yields a benefit $u_{P}\left(y, \theta_{P}\right)$ to the producer but causes an environmental damage $-u_{A}\left(y, \theta_{A}\right)$ to an agent (e.g. the local population). The producer compensates the agent for the environmental damage with a transfer $t\left(\theta_{P}, \theta_{A}\right)$ contingent to the benefit of pollution $\theta_{P}$ and its $\operatorname{cost} \theta_{A}$ (both are private information). A regulator wants to implement the ex post efficient levels of pollution $\left\{y^{*}\left(\theta_{P}, \theta_{A}\right)\right\}$ under the "polluter pays principle" which obliges the producer to pay for the environmental damages. This principle forces the agent's ex post payoffs to be non-negative. Furthermore, suppose that the producer has an outside option to produce elsewhere with a benefit denoted $\underline{u}_{P}\left(\theta_{P}\right)$. The regulator wants also to avoid this delocalization. The ex post efficient levels might be implementable with a direct revelation mechanism (while satisfying the polluter pays principle and the producer's interim participation constraints) but not with a sequential mechanism if $\underline{u}_{P}\left(\theta_{P}\right)>E_{\theta_{A}}\left[\pi\left(y^{*}\left(\theta_{P}, \theta_{A}\right),\left(\theta_{P}, \theta_{A}\right)\right)-r_{A}\left(y^{*}\left(\theta_{P}, \theta_{A}\right), \theta_{A}\right)\right]$ for a measurable set of types $\theta_{P}$. That is if the producer's outside option sometimes exceeds the total surplus net of the agent's informational rent at the interim stage. 


\section{References}

[1] Crémer, J. and M. Riordan (1985) "A Sequential Solution to the Public Goods Problem," Econometrica 53(1), 77-84.

[2] Fudenberg, D. And J. Tirole (1991) Game Theory. Mit Press.

[3] Holmström, B. and R. B. Myerson (1983) "Efficient and Durable Decision Rules with Incomplete Information," Econometrica 51, 1799-1819.

[4] Mas-Colell, A., M.D. Whinston, And J.R. Green (1995) Microeconomic Theory. Oxford University Press.

[5] Maskin, E. and J. Tirole (1990) "The Principal-Agent Relationship with an Informed Principal: The Case of Private Values," Econometrica 58, 379-409.

[6] Mirrlees, J. (1971) "An Exploration in the Theory of Optimal Income Taxation," Review of Economic Studies 38, 175-208.

[7] Mookherjee, D. And S. Reichelstein (1992) "Dominant Strategy Implementation of Bayesian Incentive Compatible Allocation Rules," Journal of Economic Theory 56, 378-399.

[8] Sappington, D. (1983) "Limited Liability Contracts Between Principal and Agent," Journal of Economic Theory 29, 1-21. 